\title{
The therapeutic landscape of advanced melanoma
}

\author{
Erika Richtig
}

Received: 29 January 2020 / Accepted: 10 March 2020 / Published online: 15 April 2020

(C) The Author(s) 2020

\begin{abstract}
Summary The therapeutic landscape of advanced and metastatic melanoma has changed dramatically in the last ten years. Targeted therapies as well as checkpoint inhibitors and oncolytic viruses have launched a broad revolution within this field. First presented at ASCO 2011, changes in melanoma treatment giving "light at the end of the tunnel" have also changed the treatment of many other tumor entities. So oncologists all over the world can offer their patients these treatment options with higher efficacy than we ever had. But despite all optimism we are still losing about half of our patients with metastatic melanoma along the way. In this short review the therapeutic landscape of advanced melanoma is described.
\end{abstract}

Keywords Melanoma - Checkpoint inhibitor · Targeted therapy · Oncolytic virus · Brain metastasis

\section{Immune checkpoint inhibitors}

Programmed cell death protein (PD-1) inhibitors are nowadays the backbone of most melanoma treatments, in many cases as the first-line treatment option. Pembrolizumab and nivolumab in treatment-naïve patients show overall survival of over $40 \%$ at 5 years. Better data were presented for the combination of the cytotoxic T-lymphocyte-associated Protein(CTLA)-4 antibody in combination with nivolumab at ESMO 2019 in Barcelona by James Larkin et al. [1]. In this study unresectable or metastatic melanoma patients were randomized 1:1:1 in the first arm of ipilimumab three milligram per kilo-

\section{E. Richtig (ه)}

Department of Dermatology, Medical University of Graz, Auenbruggerplatz 8, $8036 \mathrm{Graz}$, Austria

erika.richtig@medunigraz.at gram every 3 weeks in combination with nivolumab one milligram per kilogram for four doses followed by nivolumab three milligram per kilogram every 2 weeks. The second arm was nivolumab three milligram per kilogram every 2 weeks with ipilimumabmatched placebo and the third arm was ipilimumab three milligram per kilogram every 3 weeks for four doses with nivolumab-matched placebo. Overall survival for those patients treated with ipilimumab and nivolumab at the 5-year follow-up were $52 \%$ compared to nivolumab $44 \%$ and ipilimumab $26 \%$, giving a hazard ratio of 0.83 . The median progression-free survival (PFS) for ipilimumab and nivolumab was 11.5 months, nivolumab 6.9 months and ipilimumab 2.9 months. Patients with BRAF-mutated melanoma (V600) performed even better with overall survival rates of $60 \%$ in the ipilimumab and nivolumab arm at the 5-year follow-up compared to nivolumab (46\%) and ipilimumab (30\%). In BRAF wild-type patients the difference between the study arms were less giving overall survival of $48 \%$ for ipilimumab and nivolumab patients compared to $43 \%$ for nivolumab patients and $25 \%$ for ipilimumab patients. The improved overall survival of ipilimumab and nivolumab was independent of baseline PDL-1 expression and baseline LDH levels. The best overall response rates for ipilimumab and nivolumab was $58 \%$ followed by nivolumab $45 \%$ and ipilimumab $19 \%$. In the intent-to-treat (ITT) population median duration of response was not reached in either the ipilimumab and nivolumab arm or in the nivolumab arm. A higher proportion of patients were alive and treatment free at 5 years in the nivolumab and ipilimumab arm (74\%) compared to nivolumab (58\%) and ipilimumab (45\%).

The KEYNOTE-006 study answered the question about the outcome of the patients treated with pembrolizumab for 2 years where the treatment was stopped at this time point. These follow-up data 
after 2 years after cessation of treatment were presented at ASCO 2018 by Georgina Long et al. showing that a high percentage patients with complete remission remained in complete remission. Over $80 \%$ of patients with a partial remission remained in partial remission and even more than $50 \%$ of patients with a stable disease remained stable after stopping treatment. These data were amended by the 5 -year followup data by Robert $\mathrm{C}$ et al. [2].

The results regarding salvage therapy after failure from anti PD-1 single agent treatment were presented by Weichenthal et al. at ASCO 2019. The study showed that ipilimumab and nivolumab after failure of PD-1 treatment had 19\% objective remissions and $44 \%$ disease control rates. These results were comparable to the re-challenge of BRAF and MEK inhibitors with 22\% objective remissions and $50 \%$ disease control rates. Other treatment options (e.g., chemotherapy, T-VEC) showed better objective remissions and disease control rates than ipilimumab monotherapy [3].

Ongoing combination studies with PD-1 antibodies in combination with LAG-3 antibodies, NKTR 214 and other checkpoint inhibitors are focusing on better response rates and less toxicity.

\section{Brain metastases}

Brain metastases are a frequent problem in metastatic melanoma patients as melanoma is one of the most frequent tumors that metastasize to the brain. BRAF and MEK inhibitors as well as checkpoint inhibitors have been shown to cross the blood-brain barrier and to be able to achieve fascinating response rates. Thus, whole-brain radiation treatment has become less frequent giving space to stereotactic radiotherapy-in some cases applied only to "stimulate the immune system" before systemic treatment. Longterm outcomes from the randomized phase II study of nivolumab or nivolumab plus ipilimumab in patients with melanoma brain metastases were presented at ESMO 2019 by Georgina Long [4]. In asymptomatic patients intracranial response was achieved in $51 \%$ of patients in the nivolumab and ipilimumab arm and $20 \%$ in the nivolumab arm. In treatmentnaïve patients response rates were even better in the nivolumab and ipilimumab arm (59\%). The median intracranial progression-free survival was 5.4 months for nivolumab and ipilimumab compared to 2.5 months for nivolumab. Symptomatic patients showed median PFS of 2.6 months but only $6 \%$ intracranial responses. Overall survival rates achieved after 3 years in the nivolumab and ipilimumab arm are $49 \%$ compared to $42 \%$ in the nivolumab arm and $19 \%$ in the nivolumab arm with symptomatic patients. Intracranial and extracranial responses were mostly concordant. Interestingly activity of nivolumab plus / minus ipilimumab was low after BRAF/MEK inhibitors, after multiple modality therapies and in patients with leptomeningeal/symptomatic intracranial melanoma.

\section{Targeted therapies}

Targeted therapies with BRAF and MEK inhibitors show impressing tumor responses in a short time and are used in patients where quick response is urgently needed.

The 5-year analysis of dabrafenib plus trametinib in patients with BRAF V-600 mutated melanoma was presented at ASCO 2019 by Paul Nathan (pooled analysis of Combi-v and Combi-d) [5]. The 5-year PFS was 19\% (median PFS 11.1 months). In patients with normal LDH levels PFS at 5 years was $25 \%$ compared to $8 \%$ in patients with elevated LDH levels. The 5-year overall survival was $45 \%$. Patients with normal LDH levels achieved $43 \%$ and patients with normal LDH and at less than 3 organ sites showed even $54 \%$ overall survival (Robert et al. [2]). At ASCO 2019, Liszkay et al. presented the 4-year data of the Columbus study with encorafenib and binimetinib [6]. Overall survival of the combination with $450 \mathrm{mg}$ encorafenib and binimetinib was $39 \%$ compared to $25 \%$ of vemurafenib; progression-free survival was $25 \%$ of the Combo 450 versus $12 \%$ vemurafenib.

Different triple combinations with BRAF and MEK inhibitors in combination with anti-PD-1 and antiPDL-1 antibodies are still under investigation. Updated efficacy and safety from parts 1 and 2 of combi-i study (anti-PD-1 antibody spartalizumab in combination with dabrafenib and trametinib in previously untreated patients with advanced BRAF V600-mutant melanoma) were described by Georgina Long et al. in a poster presentation at ASCO 2019 [7]. More than $40 \%$ of patients treated with spartalizumab + dabrafenib + trametinib had confirmed complete responses, ongoing at the time of data cutoff in $67 \%$ (10 of 15) of patients. Of the patients with CR, 20\% (3 of 15) had elevated baseline LDH levels. The median PFS was 23.7 months and 10.7 months in patients with elevated baseline LDH levels. Grade $\geq 3$ AEs occurred in $78 \%$ of patients, and AEs leading to discontinuation of all 3 study drugs occurred in 6 (17\%) patients.

Data of a phase III randomized study presented at ESMO 2019 comparing cobimetinib and atezolizumab versus pembrolizumab did not meet its primary endpoint of PFS [8]. The combination of cobimetinib plus atezolizumab did not demonstrate an improvement in PFS compared to pembrolizumab monotherapy in patients with BRAF V-600 wild-type locally advanced or metastatic melanoma.

\section{T-VEC}

Oncolytic viruses aim to selectively infect tumor cells and are able to lyse them without harming normal tissues. Talimogene laherparepvec (T-VEC) is 
based on a HSV-1 virus and is licensed for stage IIIB, IIIC and IV Mla with no bone, brain, lung or other visceral metastases. The Optim study comparing T-VEC intralesional to subcutaneous GM-CSF (stage IIIB-IVM1c melanoma) showed durable response rates and best overall responses of T-VEC with $40.5 \%$ compared to $2.3 \%$ in the GM-CSF arm in the subpopulation leading to approval in stage IIIB/IIIC and IV Mla. At ESMO 2019 Middleton et al. presented a poster with a retrospective analysis of OPTiM assessed T-VEC in patients with unresectable AJCC 7 stage IIIB/C melanoma who had locoregional disease, including intralymphatic local, satellite, and regional cutaneous/subcutaneous metastases as the site of first recurrence following primary surgery [9]. T-VEC versus GM-CSF led to objective response rates of $56 \%$ vs $1 \%$, CR rates of $24 \%$ vs $0 \%$, and durable response rates of $34 \%$ vs $0 \%$. Median overall survival was not reached with T-VEC versus 25 months with GM-CSF. The locoregional subpopulation experienced higher T-VEC efficacy versus the entire study population.

\section{Take home messages}

Checkpoint inhibitors, targeted therapies and T-VEC as oncolytic virus have dramatically changed the therapeutic landscape of locally advanced and metastatic melanoma. Despite these advances, the choice of which best treatment option for the individual patient still remains with the treating physician.

Funding Open access funding provided by Medical University of Graz.

Conflict of interest E. Richtig declares speakers fees, congress supports, and honoraria for advisory boards from Almirall, Amgen, Astra Zeneca, BMS, GSK, MSD, Merck, Novartis, Pierre Fabre, Roche, and Sanofi.

Open Access This article is licensed under a Creative Commons Attribution 4.0 International License, which permits use, sharing, adaptation, distribution and reproduction in any medium or format, as long as you give appropriate credit to the original author(s) and the source, provide a link to the Creative Commons licence, and indicate if changes were made. The images or other third party material in this article are included in the article's Creative Commons licence, unless indicated otherwise in a credit line to the material. If material is not included in the article's Creative Commons licence and your intended use is not permitted by statutory regulation or exceeds the permitted use, you will need to obtain permission directly from the copyright holder. To view a copy of this licence, visit http://creativecommons.org/licenses/by/4.0/.

\section{References}

1. Larkin, J and al.: Five-Year Survival Outcomes with combined Nivolumab and Ipilimumab in advanced melanoma. NEngl J Med 2019;381:1535-1546.

2. Robert C, et al. Five-year outcomes of Dabrafenib plus Trametinib in metastatic melanoma. NEnglJ Med. 2019;15; 381(7):626-36. Aug.

3. Weichenthal M, et al. Salvage therapy after failure from anti-PD-1 single agent treatment: A Study by the German ADOReg melanoma registry.. https://meetinglibrary.asco. org/record/174824/abstract. Accessed 26.03.2020.

4. Long, G et al.: Long-term outcomes from the randomized $\mathrm{Ph} 2$ study of Nivolumab or + Ipilimumab in patients with melanomabrain metastases: Anti-PD1 Brain Collaboration (The ABC Trial). Annals of Oncology (2019) 30 (suppl_5): v533-v563. https://doi.org/10.1093/annonc/mdz255

5. Nathan P, et al. Five-year analysis on the long-term effects of dabrafenib plus trametinib $(D+T)$ in patients with BRAFV600-mutant unresectable or metastatic melanoma.. https:/ / meetinglibrary.asco.org/record/174754/abstract. Accessed 26.03.2020.

6. Liszkay G, et al. Update on Overall Survival in COLUMBUS: A randomized phase III trial of encorafenib (ENCO) plus binimetinib (BINI) versus vemurafenib (VEM) or ENCO in patients with BRAF V600-mutant melanoma.. https://meetinglibrary.asco.org/record/173422/abstract. Accessed 26.03.2020.

7. Long G, et al. The anti-PD-1 antibody spartalizumab (S) in combination with dabrafenib (D) and trametinib (T) in previously untreated patients (pts) with advanced BRAF V600-mutant melanoma: Updated efficacy and safety from parts 1 and 2 of COMBI-i.. https: / / meetinglibrary.asco.org/ record/174904/abstract. Accessed 26.03.2020.

8. Arance A, et al. Evaluation of combination treatment with Cobimetinib plus Atezolizumab versus Pembrolizumab in previously untreated patients with wild type BRAF V600 advanced melanoma: Primary analysis from the Phase 3 IMspire170. Trial. www.esmo.org. Ann Oncol. 2019;30 Suppl5:v906

9. Middleton, $\mathrm{M}$ et al.: Efficacy of talimogene laherparepvec (T-VEC) in melanoma patients (pts) with locoregional (LR) recurrence, including in-transit metastases (ITM): Subgroup analysis of the phase III OPTiM study. Ann Oncol Volume 30 | Supplement 5 | October 2019 https://doi.org/ 10.1093/annonc/mdz255|v547.

Publisher's Note Springer Nature remains neutral with regard to jurisdictional claims in published maps and institutional affiliations.

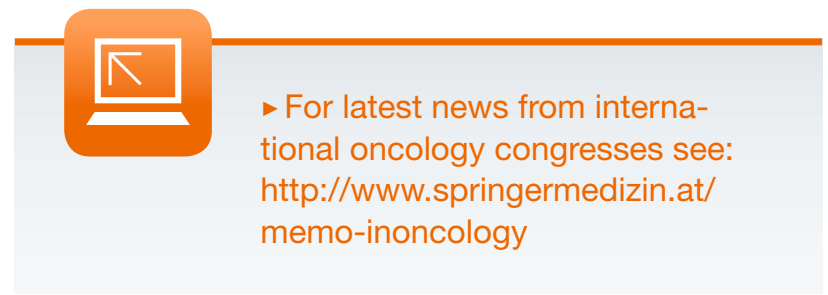

